\title{
Alternativas terapéuticas para el síndrome de apnea e hipoapnea obstructiva del sueño en niños con anomalías intermaxilares sagitales o transversales: Revisión narrativa.
}

\section{Therapeutic alternatives for obstructive sleep apnea syndrome in children with sagittal and transverse intermaxillary anomalies: Narrative review.}

\author{
Trinidad Droppelmann-Muñoz ${ }^{1}$, Constanza Carmash-Kretschmar ${ }^{1 *}$, María Ignacia Zursiedel-Puentes ${ }^{1}$, \\ Vicente Traub-Valdés ${ }^{1}$, Constanza Valdés-Kufferat ${ }^{2}$.
}

1. Práctica Privada, Univesidad de Los Andes. Santiago. Chile.

2. Área de Trastornos Temporomandibulares y Dolor Orofacial, Facultad de Odontología, Universidad de Los Andes, Santiago. Chile.

* Correspondencia Autor: Constanza Carmash | Dirección: Los Algarrobos 2304, Las Condes, Santiago, Chile. | Teléfono: +569 8338 0698. | E-mail: cvcarmash@miuandes.cl

Trabajo recibido el 20/04/2019.

Trabajo revisado 13/06/2020

Aprobado para su publicación el 16/08/2020

\section{RESUMEN}

Introducción: El síndrome de apnea e hipoapnea obstructiva del sueño corresponde a un trastorno respiratorio del sueño muy prevalente en niños. Muchas alternativas terapéuticas buscan controlar los factores desencadenantes y la progresión de los signos y síntomas. El objetivo de esta revisión es describir los efectos de los tratamientos para síndrome de apnea e hipoapnea del sueño en niños con anomalías intermaxilares sagitales o transversales. Material y método: Se realizó una búsqueda electrónica en las bases de datos: PubMed, Epistemónikos, EBSCO, TripDataBase y The Cochrane Library, con las palabras clave: "Obstructive sleep apnea", "Obstructive sleep apnoea", "Sleep apnea syndrome", "Sleep apnoea syndrome", "Sleep apnea", "Sleep apnoea", "hypoapnea", "OSA", "Treatment", "Therapy", "Children", "Pediatric", "Craniofacial abnormalities", "Craniofacial anomalies", "Craniofacial abnormality", "Orthodontic" y una búsqueda retrógrada en los textos seleccionados. Resultados: Se incluyeron 23 artículos; 12 ensayos clínicos, 6 revisiones sistemáticas, 1 estudio observacional y 4 revisiones narrativas. Conclusión: Los tratamientos ortopédicos para el síndrome de apnea e hipoapnea son la expansión rápida maxilar y dispositivos de avance mandibular. No se encontró suficiente evidencia para determinar que estos dispositivos resuelvan completamente el síndrome, pero disminuyen el índice de apnea e hipoapnea y sus signos y síntomas..

PALABRAS CLAVE:

Apnea obstructiva del sueño; Terapia; Niño; Deformidad dentofacial; Pediátrico; tratamiento.

Int. J. Inter. Dent Vol. 14(2); 165-172, 2021.

\section{ABSTRACT}

Introduction: Obstructive sleep apnea and hypoapnea syndrome refers to a respiratory sleep disorder with an increased prevalence among children. There are many therapeutic alternatives, focused on controlling trigger factors and the signs and symptoms progression. The objective of this review is to describe the effects of the available treatments for sleep apnea and hypoapnea syndrome in children with sagittal and transverse intermaxillary anomalies. Materials and Method: An electronic search was performed in PubMed database, Epistemonikos, EBSCO, Tripdatabase, and The Cochrane Library, using the keywords "Obstructive sleep apnea", "Obstructive sleep apnoea", "Sleep apnea syndrome", "Sleep apnoea syndrome", "Sleep apnea", "Sleep apnoea", "hypoapnea", "OSA", "Treatment", "Therapy", "Children", "Pediatric", "Craniofacial abnormalities", "Craniofacial anomalies", "Craniofacial abnormality" and "Orthodontic". Also, a retrieval search in the selected articles references was performed. Results: 23 articles were included; 12 clinical trials, 6 systematic reviews, 1 observational study and 4 narrative reviews. Conclusion: There are two types of orthopedic treatment for sleep apnea and hypoapnea syndrome in children with sagittal and transverse intermaxillary anomalies: rapid maxillary expansion and mandibular advancement devices. There was not enough evidence to determine that these devices achieve the syndrome's complete resolution. An important decrease in the apnea and hypoapnea index and signs and symptoms were observed.

KEY WORDS:

Obstructive sleep apnea; Therapy; Child; Dentofacial deformity; Pediatric;Treatment.

Int. J. Inter. Dent Vol. 14(2); 165-172, 2021. 


\section{INTRODUCCIÓN}

Chile está entre los ocho países con mayor privación del sueño en escolares y adolescentes. La calidad del sueño es fundamental para mantener un comportamiento adecuado y un buen rendimiento durante el día, permite la producción de actividades neurovegetativas que estimulan la reparación y mantención del organismo. Es por esto que las alteraciones del sueño en los niños generan problemas en el desarrollo y el crecimiento ${ }^{(1,2)}$.

El síndrome de apnea e hipoapnea obstructiva del sueño (SAHOS) corresponde a un trastorno respiratorio y es uno de los más frecuentes en la población escolar. Es muy importante el tratamiento precoz de este síndrome, existiendo múltiples alternativas, tales como la adenotonsilectomía, fármacos antiinflamatorios, dispositivos de presión positiva continua en la vía aérea (CPAP) y aparatos ortodóncicos intraorales, tales como el expansor maxilar y los dispositivos de avance mandibular $(2,3)$

Es importante mencionar que cuando se habla de hipoapnea, se refiere a que existe una disminución mayor al $50 \%$ del flujo aéreo nasobucal a diferencia de la apnea que es la remisión total del flujo aéreo. Es de acá de donde se desprenden los términos SAHOS y SAOS ${ }^{(4)}$

EI SAHOS es un trastorno de carácter multifactorial, y la elección de su tratamiento va a depender de cada caso particular enfocándose en los factores etiológicos que lo provocan ${ }^{(3,5)}$.

Por otra parte, las anomalías intermaxilares pueden producir una mayor oclusión de la vía aérea, aumentando así la resistencia al paso del aire y, por consiguiente, generando un mayor esfuerzo respiratorio, lo que predispone al desarrollo de $\mathrm{SAHOS}^{(6)}$.

En el sentido transversal, la compresión del maxilar determina que exista una disminución del diámetro de las vías aéreas superiores, aumentando la resistencia nasal al paso del aire, reduciendo el flujo de aire. Por otro lado, en sentido sagital, una mandíbula retrognática hará que la lengua se ubique en una posición más posterior, reduciendo así el espacio disponible de la vía aérea superior, disminuyendo el flujo de aire durante el sueño(7).

Según lo expuesto anteriormente, se puede entender la importancia de un diagnóstico y tratamiento oportuno del SAHOS en niños, para así evitar el progreso y persistencia de la enfermedad debido a sus importantes consecuencias a nivel sistémico, neurocognitivo y social en el paciente. Por lo tanto, es importante estudiar los distintos mecanismos terapéuticos dependiendo de los factores etiológicos que participan en e curso del síndrome, tal y como son las anomalías intermaxilares sagitales y transversales ${ }^{(8)}$.

El objetivo de esta revisión es describir los efectos de los distintos tratamientos que existen actualmente para el síndrome de apnea e hipoapnea obstructiva del sueño en niños con anomalías intermaxilares sagitales o transversales.

\section{MATERIAL Y MÉTODO}

Se realizó una búsqueda electrónica en las bases de datos: PubMed, Epistemónikos, EBSCO, TripDataBase y Cochrane. Se utilizaron las palabras clave: "Obstructive sleep apnea", "Obstructive sleep apnoea", "Sleep apnea syndrome", "Sleep apnoea syndrome", "Sleep apnea", "Sleep apnoea”, "Hypoapnea”, "OSA", “Treatment”, "Therapy”, "Children”, "Pediatric", "Craniofacial abnormalities", "Craniofacial anomalies", "Craniofacial abnormality", "Orthodontic" y los operadores booleanos AND y OR. Del total de artículos se hizo una primera selección por título y resumen, y luego por lectura del texto completo. Se realizó también una búsqueda manual retrógrada de la bibliografía de artículos seleccionados. Se incluyeron publicaciones en inglés y español, sin límite de año de publicación, que cumplieran con los siguientes criterios:

\section{Criterios de inclusión}

- Revisiones sistemáticas, revisiones narrativas, estudios observacionales y ensayos clínicos.

- Estudios realizados en humanos menores de 18 años.

- Pacientes con anomalías sagitales y/o transversales

\section{Criterios de exclusión:}

- Reportes de casos y opiniones de expertos.

- Estudios realizados en pacientes con alteraciones neurológicas.

- Estudios realizados en pacientes con alteraciones craneofaciales asociadas a síndromes.

Se analizó el nivel de evidencia y grado de recomendación de los estudios con la propuesta del Centre for Evidence-Based Medicine (CEBM) de Oxford. La calidad de reporte de los estudios incluidos fue evaluada con las pautas PRISMA para revisiones sistemáticas, STROBE para estudios observacionales y CONSORT para ensayos clínicos. Según el puntaje obtenido los estudios fueron clasificados con muy buena, buena, regular o mala calidad de reporte. El riesgo de sesgo fue estimado para cada artículo seleccionado basándose en la pauta para revisión de sesgo de The Cochrane Collaboration. Para la evaluación de aspectos éticos se tomaron en cuenta la presencia de consentimiento informado, aprobación de algún comité de ética, confidencialidad de antecedentes, declaración de ausencia de conflicto de intereses por parte de los autores y la descripción de posibles riesgos o efectos colaterales que sean más significantes que los posibles beneficios.

\section{RESULTADOS}

Se encontraron 848 artículos, de los cuales se preseleccionaron 125 por título y resumen, luego se eliminaron los duplicados quedando 87 artículos y se agregaron 11 mediante búsqueda retrógrada, quedando 98 artículos a los cuales se les aplicaron los criterios de inclusión y exclusión mencionados, quedando 23 artículos que cumplían con estos criterios. Estos corresponden a: 12 ensayos clínicos, 6 revisiones sistemáticas, 1 estudio observacional y 4 revisiones narrativas. 13 estudios evaluaron la expansión rápida maxilar (ERM), 6 estudios evaluaron los dispositivos de avance mandibular (DAM) y los 4 restantes evaluaron ambos tratamientos: ERM y DAM.

\section{DISCUSIÓN}

\section{Expansión rápida maxilar (ERM):}

Los niños con deficiencia transversal del maxilar presentan una resistencia nasal al flujo de aire. El estudio de Iwasaki et al. reportó un aumento del ancho nasal luego de la ERM, lo que generó un alivio de la obstrucción nasal de un $66,7 \%$ de los pacientes tratados ${ }^{(9)}$

Por otro lado, Oliveira De Felippe et al. reportaron un aumento del volumen nasal en un $18 \%$ luego de la ERM generando una disminución del $25,5 \%$ de la resistencia nasal $(10,11)$.

En pacientes Clase II esqueletal con retrusión mandibular, ocurre un reposicionamiento anterior de la lengua después de la ERM, lo que contribuye al aumento de la permeabilidad de las vías respiratorias superiores $^{(12,13)}$.

Además, es posible que el mejor posicionamiento de los dientes superiores luego del tratamiento estimule a la mandíbula a lograr una posición más anterior a medida que va creciendo(14).

Con respecto a los ensayos clínicos que evaluaron efectividad de la ERM según las variaciones de índice de apnea e hipoapnea (IAH) inicial y final, se encontró gran heterogeneidad en los resultados, en que se describen disminuciones del IAH que varían de 2,56 ev/hr - 12 ev/hr. Esta heterogeneidad, también reportada en las revisiones sistemáticas evaluadas, se puede deber a los distintos rangos de edad de inicio de tratamiento de los niños, ya que los cambios óseos transversales son más favorables cuando el tratamiento se inicia antes del peak de crecimiento puberal ${ }^{(12,14-21)}$.

Por otro lado, la diferencia en los resultados también se puede explicar por la presencia o ausencia de hipertrofia adenotonsilar. Uno de los ensayos clínicos comparó el uso de ERM previo y posterior a la adenotonsilectomia, mostrando que los pacientes sin hipertrofia adenotonsilar al momento de la expansión, disminuyeron el IAH en un $88,75 \%$ alcanzando niveles compatibles con salud (IAH < 1ev/hr). En cambio, los pacientes con ERM previa a la cirugía tuvieron una disminución del IAH de un $53,8 \%$, de los cuales sólo un paciente de quince logró la resolución de la enfermedad ${ }^{(17)}$.

Cuatro ensayos clínicos evaluaron los cambios en los signos y síntomas de los pacientes con SAHOS sometidos a EMR. En general, estos reportaron mejoras significativas en cuanto a somnolencia diurna ronquidos y descanso durante el sueño ${ }^{(10,19,22,23)}$.

Katyal et al. evaluaron los cambios en la calidad de vida de niños que recibieron tratamiento con ERM. Luego del tratamiento, se mostró una mejora del $14 \%$ de calidad de vida de los pacientes del grupo de alto riesgo, pero un empeoramiento del $1 \%$ de calidad de vida del grupo de bajo riesgo. Este resultado negativo se adjudicó a que el aparato puede llevar a una sensación de menor volumen intraoral y porque afecta el mantenimiento de la higiene bucal y el habla, alterando la calidad de la vida de estos pacientes ${ }^{(22,23)}$

Es importante recalcar que se reportaron muchos casos de SAHOS con resolución incompleta. Esto se puede atribuir al carácter multifactorial de este síndrome, en que un único tratamiento no es suficiente para lograr la resolución completa, siendo necesario un enfoque multidisciplinario. Camacho et al. proponen que en pacientes sometidos a adenotonsilectomía y ERM, que aún presenten SAHOS residual, es importante considerar otros sitios de posible obstrucción durante el sueño como lo es el colapso de la epiglotis, supraglotis o base de la lengua(12, 14, 15, 17-20). 
Seis estudios mostraron una estabilización de los resultados logrados con ERM a largo plazo, no existiendo cambios significativos en IAH, signos y síntomas y parámetros del sueño en el tiempo(10, 12, 14, 16, 19, 24).

Otros estudios evaluaron parámetros del sueño y cambios en la saturación de oxígeno arterial en la polisomnografía (PSG). Miano et al. reportaron una mejora significativa en el tiempo total de sueño, tiempo en cama y despertares por hora. No hubo cambios significativos para el porcentaje de sueño REM (Rapid Eye Movement), tiempo de latencia del inicio del sueño y eficiencia del sueño. Estos resultados coincidieron con otros estudios similares ${ }^{(18)}$.

En cuanto a la variación del menor valor de saturación arterial de oxígeno, los resultados fueron significativos sólo en el estudio de Guilleminault et al. en que hubo un aumento del $3,4 \%$ luego del tratamiento con ERM en pacientes que presentaban hipertrofia amigdalina y un aumento del $2,8 \%$ luego de la expansión en pacientes que ya habían sido sometidos a cirugía ${ }^{(17,19,20)}$.

Por otro lado, una revisión narrativa incluyó estudios que reportaron una relación entre la compresión maxilar y las enfermedades respiratorias infecciosas, rinitis alérgica y asma, observando que los pacientes con mordida cruzada tenían tres veces más enfermedades. Luego del tratamiento con ERM disminuyeron considerablemente debido a que se favoreció la respiración nasal(25).

Con respecto a otros efectos reportados en los estudios, luego de la terapia con ERM, Miano et al. evaluaron la presencia de hipertrofia tonsilar antes y después del tratamiento. Luego de la terapia hubo una disminución en la severidad de la condición e incluso resolución completa, debido a la ausencia de respiración bucal, la que es un factor irritante desencadenante de hipertrofia tonsilar ${ }^{(18)}$

\section{Dispositivos de avance mandibular (DAM):}

Los DAM son aparatos funcionales que buscan avanzar la posición de la mandíbula, llevando también a un posicionamiento anterior de la base de los músculos de la lengua. Esto genera una tracción del hueso hioides, el que se eleva por los músculos y ligamentos, mejorando la morfología de las vías respiratorias. Estos cambios permiten un aumento en el diámetro de las vías respiratorias y del espacio aéreo superior en la zona velofaríngea, debido a un aumento en su dimensión lateral. Por otro lado, se ha postulado que los DAM generarían una estimulación de los músculos dilatadores de las vías aéreas superiores, lo que llevaría a su estabilización ${ }^{(13,15,26-28)}$.

Un estudio comparó el tratamiento con DAM versus el no tratamiento del SAHOS, logrando una disminución de al menos $50 \%$ del $\mathrm{IAH}$ en $64,28 \%$ de los pacientes. Además, se observó una disminución significativa de los síntomas diurnos y nocturnos ${ }^{(29)}$.

Otro ensayo clínico que evaluó el IAH, logró una disminución de éste en un promedio de $75,9 \%$ luego del tratamiento con DAM, a la vez que el menor valor de saturación de oxígeno arterial aumentó en un $15,85 \%(30)$.

Sin embargo, en ningún estudio se logró llegar a un $\mathrm{IAH}<1$, compatible con la mejora del SAHOS. Por ende, al igual que lo propuesto con la ERM, los DAM como único tratamiento no serían suficientes para la resolución completa de la enfermedad, debido a su carácter etiológico multifactorial(15, 26, 28, 29, 31).

Villa et al. reportaron una disminución en la hipertrofia tonsilar del $66,7 \%$ de los pacientes del grupo intervenido con DAM. Esto se puede atribuir a que, luego del tratamiento, se favorece la respiración nasal por sobre la bucal, disminuyendo así las infecciones y las irritaciones a las tonsilas palatinas ${ }^{(29)}$.
Ningún estudio especifica si la PSG final fue realizada con el DAM instalado, esto es importante ya que si se quiere evaluar cambios esqueletales permanentes de avance mandibular, se deber realizar el examen sin el dispositivo en boca.

\section{Tratamiento con ERM y DAM simultáneo:}

Cozza et al. trataron a sus pacientes con un DAM al cual se le añadió un tornillo en centro del maxilar que permite una expansión al mismo tiempo que se logra la posición anterior de la mandíbula, obteniendo una disminución promedio del IAH de un 46,9\% además de mejoras en los síntomas diurnos y nocturnos ${ }^{(30)}$.

Es de gran importancia mencionar que a pesar de que la mayoría de los estudios incluidos tenían buena calidad de reporte, el ideal para evaluar estos tratamientos son los ensayos clínicos controlados aleatorizados con un grupo control que no reciba tratamiento. De estos se encontró tan sólo uno en la literatura que cumpliera con los criterios de inclusión y exclusión. Sin este tipo de estudios no se puede saber si los niños que recibieron tratamiento podrían haber mejorado sin intervención, como resultado del crecimiento. Pero existen implicancias éticas que impiden este tipo de estudio al no realizarle un tratamiento disponible a un grupo de pacientes diagnosticados con una patología.

Dentro de las limitaciones de esta revisión se encuentra la diversidad de mecanismos diagnósticos de SAHOS en los estudios incluidos, por lo que esta falta de criterio diagnóstico estandarizado no permite establecer un medio de comparación universal de los resultados, ya que lo ideal habría sido evaluar las variaciones del IAH como resultado primario. Por otro lado, cabe destacar que no existe consenso en lo que corresponde a éxito y fracaso del tratamiento, en que algunos estudios lo evalúan de acuerdo a la resolución completa de SAHOS (IAH $<1 \mathrm{ev} / \mathrm{hr})$, mientras que otros estudios lo determinan de acuerdo a una reducción significativa de este índice. Es por esto que también se presentan dificultades al describir la eficacia de los tratamientos.

También es una limitación el que la mayoría de los estudios no menciona ni describe el tipo de dispositivo de avance mandibular o de expansión maxilar utilizado.

Por último, es importante mencionar que se necesita un mayor estudio de los efectos a largo plazo de estos tratamientos mediante PSG, con reportes de seguimiento de los pacientes luego de terminado este tratamiento hasta que alcancen la edad adulta. Esto para poder evaluar las posibles recidivas que existan y que predispongan a un empeoramiento de la condición.

\section{CONCLUSIÓN}

Existen dos tipos de tratamiento ortopédico para el SAHOS en niños con anomalías intermaxilares sagitales y transversales, la expansión rápida maxilar (ERM) y los dispositivos de avance mandibular (DAM). No se encontró suficiente evidencia para determinar que estos dispositivos alcancen la resolución completa del síndrome. Sí se observó que logran una gran disminución del índice de apnea e hipoapnea (IAH) y de los signos y síntomas que lo acompañan, ya que ayudan a mejorar la permeabilidad de la vía aérea superior durante el sueño mediante la ampliación de ésta y/o mediante la disminución de su colapso, mejorando el tono muscular de la vía aérea superior.

\section{CONFLICTO DE INTERESES}

Los autores no declaran conflicto de interés. 
Tabla 1: Resumen de artículos.

\begin{tabular}{|c|c|c|c|c|c|}
\hline $\mathbf{N}^{\circ}$ & Autores & $\begin{array}{l}\text { Tipo de } \\
\text { Estudio }\end{array}$ & Objetivo & $\begin{array}{c}\text { Tratamiento } \\
\text { evaluado }\end{array}$ & Resultados/Conclusiones \\
\hline 1 & $\begin{array}{l}\text { Carvhalo FR, } \\
\text { et al. } .^{(48)}\end{array}$ & $\begin{array}{l}\text { Revisión } \\
\text { sistemática }\end{array}$ & $\begin{array}{l}\text { Evaluar los efectos de los } \\
\text { aparatos orales o aparatos } \\
\text { ortopédicos funcionales para la } \\
\text { apnea obstructiva del sueño en } \\
\text { niños. }\end{array}$ & DAM & $\begin{array}{l}\text { Un estudio incluido que comparó un aparato } \\
\text { oral con un grupo sin tratamiento y presentó } \\
\text { resultados analizando cada grupo antes y } \\
\text { después de seis meses de seguimiento. Se } \\
\text { encontraron resultados favorables en el grupo } \\
\text { tratado en: IAH, síntomas diurnos y nocturnos. } \\
\text { Conclusiones: en la actualidad no hay suficiente } \\
\text { evidencia para afirmar que los aparatos orales } \\
\text { o los aparatos ortopédicos funcionales son } \\
\text { eficaces en el tratamiento de la apnea obstructiva } \\
\text { del sueño en niños. }\end{array}$ \\
\hline 2 & $\begin{array}{l}\text { Nazarali N, et } \\
\text { al.(49) }\end{array}$ & $\begin{array}{l}\text { Revisión } \\
\text { sistemática }\end{array}$ & $\begin{array}{l}\text { Evaluar la efectividad de los } \\
\text { aparatos de avance mandibular } \\
\text { para el tratamiento de SAHOS } \\
\text { en niños. }\end{array}$ & DAM & $\begin{array}{l}\text { Incluyó } 4 \text { artículos. Conclusiones: la evidencia } \\
\text { puede ser sugestiva de que los DAM resultan en } \\
\text { mejoras a corto plazo en las puntuaciones del } \\
\text { IAH, pero no es posible concluir que son eficaces } \\
\text { para tratar el SHAOS pediátrico. }\end{array}$ \\
\hline 3 & $\begin{array}{l}\text { Huynh NT, et } \\
\text { al. } .^{(50)}\end{array}$ & $\begin{array}{l}\text { Revisión } \\
\text { sistemática }\end{array}$ & $\begin{array}{l}\text { Investigar la eficacia del } \\
\text { avance mandibular ortopédico } \\
\text { y/o expansión maxilar rápida } \\
\text { en el tratamiento de la apnea } \\
\text { obstructiva el sueño en edad } \\
\text { pediátrica. }\end{array}$ & EMR y DAM & $\begin{array}{l}6 \text { estudios incluidos. Conclusiones: estos } \\
\text { tratamientos ortodónticos pueden ser eficaces } \\
\text { en el manejo del ronquido pediátrico y la } \\
\text { apnea obstructiva del sueño. Otros resultados } \\
\text { relacionados con la salud, como las funciones } \\
\text { neurocognitivas y cardiovasculares aún no se } \\
\text { han abordado sistemáticamente. }\end{array}$ \\
\hline 4 & $\begin{array}{l}\text { Machado- } \\
\text { Junior A, et } \\
\text { al. }{ }^{(51)}\end{array}$ & $\begin{array}{l}\text { Revisión } \\
\text { sistemática }\end{array}$ & $\begin{array}{l}\text { Realizar un metanálisis en } \\
\text { estudios de todo el mundo que } \\
\text { utilizaron EMR para tratar el } \\
\text { SAHOS en niños }\end{array}$ & EMR & $\begin{array}{l}10 \text { artículos incluidos. Conclusiones: la expansión } \\
\text { maxilar rápida en niños con SAHOS parece ser } \\
\text { un tratamiento eficaz para este síndrome. Se } \\
\text { necesitan más ensayos clínicos aleatorizados. }\end{array}$ \\
\hline 5 & $\begin{array}{l}\text { Baratieri C, } \\
\text { et al. }{ }^{(52)}\end{array}$ & $\begin{array}{l}\text { Revisión } \\
\text { sistemática }\end{array}$ & $\begin{array}{l}\text { Identificar y calificar la evidencia } \\
\text { de informes a largo plazo que } \\
\text { evaluaran los cambios en las } \\
\text { dimensiones y funciones de las } \\
\text { vías respiratorias en pacientes } \\
\text { con EMR durante el período de } \\
\text { crecimiento }\end{array}$ & EMR & $\begin{array}{l}8 \text { estudios incluidos. Las radiografías } \\
\text { posteroanteriores mostraron que el ancho } \\
\text { de la cavidad nasal aumenta. La tomografía } \\
\text { computarizada por haz de cono no mostró } \\
\text { aumentos significativos del volumen de la } \\
\text { cavidad nasal. La rinomanometría mostró } \\
\text { reducción de la resistencia nasal de las vías } \\
\text { respiratorias y aumento del flujo nasal total, y la } \\
\text { rinometría acústica detectó aumentos de área } \\
\text { transversal mínima y volumen de la cavidad } \\
\text { nasal. Conclusiones: Hay evidencia moderada de } \\
\text { que los cambios después de EMR en los niños } \\
\text { en crecimiento mejoran las condiciones para } \\
\text { la respiración nasal y los resultados se puede } \\
\text { esperar que sean estable durante al menos } 11 \\
\text { meses después de la terapia. }\end{array}$ \\
\hline 6 & $\begin{array}{l}\text { Camacho M, } \\
\text { et al. }{ }^{(53)}\end{array}$ & $\begin{array}{l}\text { Revisión } \\
\text { sistemática }\end{array}$ & $\begin{array}{l}\text { Realizar una revisión sistemática } \\
\text { de la literatura internacional } \\
\text { sobre la EMR como tratamiento } \\
\text { para SAHOS pediátrico, seguido } \\
\text { de un metanálisis de los datos } \\
\text { disponibles. }\end{array}$ & EMR & $\begin{array}{l}17 \text { estudios incluidos. Se ha observado una } \\
\text { mejoría del IAH y menor saturación de oxígeno } \\
\text { en los niños sometidos a EMR, especialmente a } \\
\text { corto plazo (<3 años de seguimiento). Ensayos } \\
\text { aleatorios y más estudios que informen datos a } \\
\text { largo plazo (seguimiento de } 3 \text { años) ayudarían } \\
\text { a determinar el efecto del crecimiento y la } \\
\text { resolución espontánea de la SAHOS. }\end{array}$ \\
\hline 7 & $\begin{array}{l}\text { Villa MP, et } \\
\text { al. }{ }^{(54)}\end{array}$ & $\begin{array}{l}\text { Ensayo } \\
\text { Clínico } \\
\text { Controlado } \\
\text { Aleatorizado }\end{array}$ & $\begin{array}{l}\text { Evaluar la utilidad clínica y la } \\
\text { tolerancia de un dispositivo } \\
\text { oral personalizado de } \\
\text { posicionamiento de la mandíbula } \\
\text { en el tratamiento de SAHOS en } \\
\text { niños con maloclusión. }\end{array}$ & DAM & $\begin{array}{l}\text { Un grupo de } 19 \text { participantes fue asignado } \\
\text { aleatoriamente a un ensayo de } 6 \text { meses con } \\
\text { un aparato oral, el resto fue el grupo control } \\
4 \text { sujetos tratados y } 5 \text { controles se perdieron } \\
\text { en el seguimiento. Resultados: después del } \\
\text { ensayo se vio que los sujetos tratados tenían } \\
\text { IAH significativamente más bajos, mientras que } \\
\text { en el grupo control estos valores se mantuvieron } \\
\text { casi intactos. Se vio que el aparato había logrado } \\
\text { la disminución de los síntomas en } 7 \text { de los } \\
14 \text { pacientes tratados y en } 7 \text { pacientes había } \\
\text { resuelto los síntomas, mientras que los controles } \\
\text { continuaron presentando síntomas. }\end{array}$ \\
\hline
\end{tabular}


Tabla 1: Resumen de artículos. (continuación)

\begin{tabular}{|c|c|c|c|c|c|}
\hline $\mathbf{N}^{\circ}$ & Autores & $\begin{array}{l}\text { Tipo de } \\
\text { Estudio }\end{array}$ & Objetivo & $\begin{array}{c}\text { Tratamiento } \\
\text { evaluado }\end{array}$ & Resultados/Conclusiones \\
\hline 8 & $\begin{array}{l}\text { Rizk S, et } \\
\text { al. }{ }^{(55)}\end{array}$ & $\begin{array}{l}\text { Ensayo } \\
\text { Clínico } \\
\text { Controlado } \\
\text { no } \\
\text { Aleatorizado }\end{array}$ & $\begin{array}{l}\text { Evaluar los cambios en la } \\
\text { posición ósea del hioides, el } \\
\text { volumen orofaríngeo y las } \\
\text { dimensiones en los planos } \\
\text { transversales y sagitales } \\
\text { después del tratamiento de los } \\
\text { pacientes esqueléticos de Clase } \\
\text { Il con aparatos funcionales } \\
\text { seguidos de tratamiento con } \\
\text { DAM. }\end{array}$ & DAM & $\begin{array}{l}\text { Se compararon } 20 \text { pacientes Clase II tratados } \\
\text { con DAM seguido de aparatos fijos, con una } \\
\text { muestra de control no tratada por etapa de } \\
\text { maduración de vértebras cervicales. El volumen } \\
\text { de la vía aérea orofaríngea, las dimensiones } \\
\text { de las vías respiratorias y la posición antero } \\
\text { posterior del hueso hioides aumentaron } \\
\text { significativamente con el tratamiento funcional de } \\
\text { los dispositivos. }\end{array}$ \\
\hline 9 & $\begin{array}{l}\text { Guilleminault } \\
\mathrm{C} \text {, et al.(56) }\end{array}$ & $\begin{array}{l}\text { Ensayo } \\
\text { Clínico Piloto }\end{array}$ & No especifica. & EMR & $\begin{array}{l}31 \text { niños, diagnosticados con SAHOS, tenían } \\
\text { presencia de complejos maxilares estrechos y } \\
\text { amígdalas hipertrofiadas. Fueron programados } \\
\text { para tener tanto adeno-amigdalectomía como EMR } \\
\text { para el cual el orden del tratamiento fue aleatorio: el } \\
\text { grupo } 1 \text { recibió la cirugía seguida por la ortodoncia, } \\
\text { mientras que el grupo } 2 \text { recibió la ortodoncia } \\
\text { seguida de la cirugía. Resultados: los niños } \\
\text { presentaron mejoría tanto de los síntomas clínicos } \\
\text { como de los resultados del PSG, pero ninguno de } \\
\text { los niños presentó resultados normales después } \\
\text { del tratamiento } 1, \text { a excepción de un caso. No hubo } \\
\text { diferencias significativas en la cantidad de mejora } \\
\text { observada independientemente del primer enfoque } \\
\text { de tratamiento. Ambos tratamientos combinados sí } \\
\text { lograban la mejora. }\end{array}$ \\
\hline 10 & $\begin{array}{l}\text { Iwasaki T, et } \\
\text { al. }{ }^{(57)}\end{array}$ & $\begin{array}{l}\text { Ensayo } \\
\text { clínico no } \\
\text { controlado }\end{array}$ & $\begin{array}{l}\text { Uso de la dinámica de fluidos } \\
\text { computacional para evaluar } \\
\text { con más precisión y clarificar la } \\
\text { cantidad de la ventilación de la } \\
\text { vía aérea nasal después de la } \\
\text { EMR. }\end{array}$ & EMR & $\begin{array}{l}\text { La medida del ancho nasal después de la EMR } \\
\text { fue significativamente mayor que previo a la } \\
\text { expansión (aprox. } 3 \mathrm{~mm} \text { ). Así como también } \\
\text { el ancho molar fue mayor después de la } \\
\text { EMR (aprox. } 5 \mathrm{~mm} \text { ). Previo a la EMR } 18 \text { de } \\
23 \text { pacientes ( } 78 \% \text { ) tenían una obstrucción } \\
\text { detectada con la reconstrucción } 3 D \text { o con la } \\
\text { dinámica de fluidos computacional. Luego de la } \\
\text { EMR, } 12 \text { de los } 18 \text { pacientes ( } 66,7 \% \text { ) que tenían } \\
\text { una obstrucción antes de la EMR, ya no tenían } \\
\text { obstrucción alguna luego de la EMR }\end{array}$ \\
\hline 11 & $\begin{array}{l}\text { Miano S, et } \\
\text { al.(58) }\end{array}$ & $\begin{array}{l}\text { Ensayo } \\
\text { clínico } \\
\text { controlado no } \\
\text { aleatorizado }\end{array}$ & $\begin{array}{l}\text { Evaluar la microestructura del } \\
\text { sueño NREM en niños con } \\
\text { SHAOS antes y después de un } \\
\text { año de tratamiento con EMR por } \\
\text { medio del patrón alterno cíclico }\end{array}$ & EMR & $\begin{array}{l}\text { Se incluyeron nueve niños con SHAOS de } 4 \text { a } \\
8 \text { años y controles normales de la misma edad. } \\
\text { Después de un año de tratamiento, el grupo } \\
\text { SAHOS mostró una mayor tiempo de sueño y en } \\
\text { cama, una reducción en el número de cambios } \\
\text { de etapa en comparación con los registros } \\
\text { basales y el IAH disminuyó significativamente. } \\
\text { Sin embargo, la microestructura del sueño y } \\
\text { los parámetros respiratorios no se recuperaron } \\
\text { completamente. }\end{array}$ \\
\hline 12 & $\begin{array}{l}\text { Villa MP, et } \\
\text { al.(59) }\end{array}$ & $\begin{array}{l}\text { Ensayo } \\
\text { clínico no } \\
\text { controlado }\end{array}$ & $\begin{array}{l}\text { Confirmar la eficacia de la } \\
\text { EMR en niños con hipertrofia } \\
\text { adenotonsilar moderada y } \\
\text { evaluar retrospectiva-mente sus } \\
\text { beneficios a largo plazo después } \\
\text { del inicio de la pubertad en un } \\
\text { grupo de niños sometidos a } \\
\text { tratamiento ortodóntico hace } 10 \\
\text { años }\end{array}$ & EMR & $\begin{array}{l}40 \text { pacientes fueron reclutados. Luego de } 12 \\
\text { meses de tratamiento con EMR } 34 / 40 \text { pacientes } \\
(85) \text { mostraron una disminución del IAH mayor } \\
\text { al } 20 \% \text { y se catalogaron como respondedores. } \\
\text { Sólo } 6 \text { de los } 40 \text { pacientes ( } 15 \% \text { ) mostraron } \\
\text { una disminución del IAH menor al } 20 \% \text { y se } \\
\text { catalogaron como no respondedores. Por otro } \\
\text { lado, el } 57,5 \% \text { de los pacientes presentó SAHOS } \\
\text { residual (IAH mayor a } 1 \text { ev/hr) después del } \\
\text { tratamiento. Catorce niños que terminaron el } \\
\text { tratamiento ortodóntico } 10 \text { años antes mostraron } \\
\text { una mejoría en los signos y síntomas. }\end{array}$ \\
\hline
\end{tabular}


Tabla 1: Resumen de artículos. (continuación)

\begin{tabular}{|c|c|c|c|c|c|}
\hline $\mathbf{N}^{\circ}$ & Autores & $\begin{array}{l}\text { Tipo de } \\
\text { Estudio }\end{array}$ & Objetivo & $\begin{array}{c}\text { Tratamiento } \\
\text { evaluado }\end{array}$ & Resultados/Conclusiones \\
\hline 13 & $\begin{array}{l}\text { Katyal V, et } \\
\text { al. }{ }^{(60)}\end{array}$ & $\begin{array}{l}\text { Ensayo } \\
\text { clínico no } \\
\text { controlado }\end{array}$ & $\begin{array}{l}\text { Evaluar la prevalencia de } \\
\text { niños con riesgo de SDB, } \\
\text { según lo identificado en un } \\
\text { entorno de ortodoncia mediante } \\
\text { cuestionarios validados, y } \\
\text { examinar las asociaciones con } \\
\text { la morfología craneofacial y de } \\
\text { las vías respiratorias superiores. } \\
\text { Un objetivo adicional fue evaluar } \\
\text { el cambio en la calidad de vida } \\
\text { relacionada con SDB para los } \\
\text { niños afectados que tienen } \\
\text { ERM para corregir una mordida } \\
\text { cruzada palatina o ensanchar un } \\
\text { maxilar inferior }\end{array}$ & EMR & $\begin{array}{l}\text { Los niños que se sometieron a una expansión } \\
\text { maxilar rápida fueron seguidos longitudinalmente } \\
\text { hasta la extracción del aparato aproximadamente } \\
9 \text { meses después, con un cuestionario de calidad } \\
\text { de vidaaApnea obstructiva del sueño- } 18 \text { repetido. } \\
\text { Los niños tratados con expansión maxilar rápida } \\
\text { mostraron una mejora promedio del } 14 \% \text { en las } \\
\text { puntuaciones de calidad de vida en el grupo de } \\
\text { alto riesgo en comparación con el grupo de bajo } \\
\text { riesgo, que mostró un ligero empeoramiento de } \\
\text { la calidad de vida de un } 1 \% \text {. }\end{array}$ \\
\hline 14 & $\begin{array}{l}\text { Villa MP, et } \\
\text { al.(61) }\end{array}$ & $\begin{array}{l}\text { Ensayo } \\
\text { clínico no } \\
\text { controlado }\end{array}$ & $\begin{array}{l}\text { El objetivo del presente estudio } \\
\text { fue evaluar el resultado del } \\
\text { tratamiento quirúrgico (AT) y } \\
\text { del tratamiento ortodóncico } \\
\text { (aplicación de la expansión } \\
\text { maxilar rápida endo-oral) en } \\
\text { niños con respiración alterada del } \\
\text { sueño, que fueron sometidos a } \\
\text { terapia quirúrgica o ortodóncica, } \\
\text { o ambos, si presentan hipertrofia } \\
\text { adenotonsilar y/o paladar } \\
\text { estrecho con maloclusiones. }\end{array}$ & EMR & $\begin{array}{l}\text { Un total de } 52 \text { sujetos cumplieron los criterios de } \\
\text { inclusión. Veinticinco niños fueron sometidos a } \\
\text { AT (grupo 1) y } 22 \text { niños fueron sometidos a EMR } \\
\text { (grupo 2). Cinco niños se sometieron a ambos } \\
\text { tratamientos (grupo 3). Después de } 1 \text { año, el IMC } \\
\text { percentil y la saturación media durante la noche } \\
\text { aumentaron en el grupo 1, mientras que el IAH y } \\
\text { el índice de excitación disminuyeron. En el grupo } \\
\text { 2, la saturación media durante la noche aumentó } \\
\text { mientras que disminuyó el IAH. Los niños del } \\
\text { grupo } 3 \text { mostraron una disminución significativa } \\
\text { en el IAH de T0 a T1. }\end{array}$ \\
\hline 15 & $\begin{array}{l}\text { Giannasi LC, } \\
\text { et al. }{ }^{(62)}\end{array}$ & $\begin{array}{l}\text { Ensayo } \\
\text { clínico piloto }\end{array}$ & $\begin{array}{l}\text { Evaluar la eficacia del dispositivo } \\
\text { de expansión maxilar rápida para } \\
\text { el tratamiento de trastornos del } \\
\text { sueño en niños }\end{array}$ & EMR & $\begin{array}{l}\text { Se observaron mejoras significativas en el } \\
\text { cansancio al despertar, humor, sello labial, } \\
\text { babeo durante el sueño, ronquido y bruxismo. } \\
\text { Conclusión: El uso de métodos no invasivos, } \\
\text { como la EMR, puede ser un tratamiento eficaz } \\
\text { para el ronquido y otros comportamientos } \\
\text { indeseables del sueño en niños. }\end{array}$ \\
\hline 16 & $\begin{array}{l}\text { Cozza P, et } \\
\text { al.(63) }\end{array}$ & $\begin{array}{l}\text { Ensayo } \\
\text { clínico no } \\
\text { controlado }\end{array}$ & $\begin{array}{l}\text { Determinar las diferencias entre } \\
\text { los niños con apnea obstructiva } \\
\text { del sueño (SAHOS) y los sujetos } \\
\text { control, e investigar los efectos } \\
\text { del tratamiento con DAM en } \\
\text { niños con SAHOS. }\end{array}$ & $\begin{array}{l}\text { EMR y DAM } \\
\text { simultáneamente }\end{array}$ & $\begin{array}{l}\text { La polisomnografía repetida, con el DAM in } \\
\text { situ, después de } 6 \text { meses de uso, reveló una } \\
\text { reducción significativa del IAH en niños con } \\
\text { SAHOS. Se encontró que el DAM reducía la } \\
\text { somnolencia diurna y mejoraba subjetivamente la } \\
\text { calidad del sueño evaluada. Los pacientes y sus } \\
\text { padres informaron un buen cumplimiento con el } \\
\text { tratamiento con DAM. }\end{array}$ \\
\hline 17 & $\begin{array}{l}\text { Zhang C, et } \\
\text { al. }^{(64)}\end{array}$ & $\begin{array}{l}\text { Ensayo } \\
\text { clínico no } \\
\text { controlado }\end{array}$ & $\begin{array}{l}\text { Investigar los efectos del DAM en } \\
\text { niños con SAHOS y retrognatia } \\
\text { mandibular. }\end{array}$ & DAM & $\begin{array}{l}\text { Un total de } 46 \text { niños diagnosticados con } \\
\text { retrognatia mandibular y SAHOS por PSG y } \\
\text { sin obesidad o hipertrofia tonsilar se reclutaron } \\
\text { para el estudio. Los resultados mostraron una } \\
\text { mejora en el perfil facial del paciente después } \\
\text { del tratamiento con el DAM. El promedio del IAH } \\
\text { disminuyó de } 14.08 \text { a } 3.39 \text { y el nivel más bajo de } \\
\text { SaO2 aumentó de } 77.78 \text { a 93.63. }\end{array}$ \\
\hline 18 & $\begin{array}{l}\text { Oliveira De } \\
\text { Felippe NL, } \\
\text { et al. }{ }^{(65)}\end{array}$ & $\begin{array}{l}\text { Ensayo } \\
\text { clínico no } \\
\text { controlado }\end{array}$ & $\begin{array}{l}\text { Determinar los si los cambios } \\
\text { en la anatomía dental maxilar } \\
\text { afecta la anatomía y función de la } \\
\text { cavidad nasal. }\end{array}$ & EMR & $\begin{array}{l}\text { Los efectos a corto plazo estadísticamente } \\
\text { significativos de EMR fueron: (1) aumento medio } \\
\text { en el área palatina, el volumen y la distancia } \\
\text { intermolar; (2) una reducción media de la } \\
\text { resistencia nasal de las vías respiratorias; y (3) } \\
\text { significativo aumento en el volumen nasal total } \\
\text { y en el área de la cavidad nasal. Resultados } \\
\text { a largo plazo fueron los siguientes: (1) el área } \\
\text { palatina media y la distancia intermolar se } \\
\text { redujeron, mientras que el volumen palatal } \\
\text { fue estable, y (2) la resistencia nasal de las } \\
\text { vías respiratorias fue estable, mientras que el } \\
\text { volumen medio de la cavidad nasal y el área } \\
\text { transversal mínima aumentaron. Adicionalmente, } \\
\text { el } 61,3 \% \text { de los sujetos reportaron mejoría } \\
\text { subjetiva en la respiración nasal. }\end{array}$ \\
\hline
\end{tabular}


Tabla 1: Resumen de artículos. (continuación)

\begin{tabular}{|c|c|c|c|c|c|}
\hline $\mathbf{N}^{\circ}$ & Autores & $\begin{array}{l}\text { Tipo de } \\
\text { Estudio }\end{array}$ & Objetivo & $\begin{array}{l}\text { Tratamiento } \\
\text { evaluado }\end{array}$ & Resultados/Conclusiones \\
\hline 19 & $\begin{array}{l}\text { Pirelli P, et } \\
\text { al. }^{\left({ }^{6}\right)}\end{array}$ & $\begin{array}{l}\text { Estudio } \\
\text { longitudinal } \\
\text { prospectivo }\end{array}$ & $\begin{array}{l}\text { Evaluar prospectivamente la } \\
\text { eficacia a largo plazo de la } \\
\text { EMR en un grupo de niños con } \\
\text { SAHOS }\end{array}$ & EMR & $\begin{array}{l}\text { Las evaluaciones clínicas anuales, incluidas los } \\
\text { exámenes odontológicos y laringológicos, junto } \\
\text { con las puntuaciones de los cuestionarios, fueron } \\
\text { consistentemente normales en el transcurso del } \\
\text { tiempo y los hallazgos de la PSG permanecieron } \\
\text { normales en el período de seguimiento de } 12 \\
\text { años. La estabilidad y el mantenimiento de la } \\
\text { expansión a lo largo del tiempo se demostró por } \\
\text { el ancho de la base del maxilar y la distancia de } \\
\text { los procesos pterigoideos medidos mediante el } \\
\text { TAC. }\end{array}$ \\
\hline 20 & $\begin{array}{l}\text { Villa MP, et } \\
\text { al. }{ }^{(67)}\end{array}$ & $\begin{array}{l}\text { Revisión } \\
\text { narrativa }\end{array}$ & No especifica & EMR & $\begin{array}{l}\text { La EMR puede aliviar los problemas respiratorios } \\
\text { mediante un incremento en las dimensiones } \\
\text { transversales del maxilar, lo que lleva a un } \\
\text { ensanchamiento de la cavidad nasal. El } \\
\text { ensanchamiento del maxilar corrige mordidas } \\
\text { cruzadas posteriores, mejora la coordinación } \\
\text { entre los arcos maxilares y mandibulares } \\
\text { previo al tratamiento ortopédico y funcional } \\
\text { de las maloclusiones clases II y III, y aumenta } \\
\text { el perímetro del arco de los pacientes con } \\
\text { discrepancias dentoalveolares. }\end{array}$ \\
\hline 21 & $\begin{array}{l}\text { Ngiam J, et } \\
\text { al. }(68)\end{array}$ & $\begin{array}{l}\text { Revisión } \\
\text { narrativa }\end{array}$ & $\begin{array}{l}\text { Discutir la relación entre el } \\
\text { desarrollo cráneo facial y } \\
\text { SAHOS en niños, con un } \\
\text { enfoque en los tratamientos } \\
\text { dentales emergentes tales } \\
\text { como EMR, dispositivos orales } \\
\text { e intervenciones quirúrgicas } \\
\text { máxilo-mandibulares, como } \\
\text { distracción osteogénica, como } \\
\text { manejo en los TRS en la } \\
\text { población pediátrica }\end{array}$ & EMR y DAM & $\begin{array}{l}\text { Se describen los tratamientos de expansión } \\
\text { maxilar rápida y dispositivos de adelantamiento } \\
\text { mandibular. Lo que es la distracción osteogénica } \\
\text { se describe para pacientes con síndromes } \\
\text { asociados }\end{array}$ \\
\hline 22 & $\begin{array}{l}\text { Kiliç N, et } \\
\text { al. }{ }^{(69)}\end{array}$ & $\begin{array}{l}\text { Revisión } \\
\text { narrativa }\end{array}$ & $\begin{array}{l}\text { Revisar los efectos de la EMR } \\
\text { en los problemas respiratorios } \\
\text { causados por la constricción } \\
\text { maxilar y la respiración bucal en } \\
\text { niños en crecimiento }\end{array}$ & EMR & $\begin{array}{l}\text { Aunque el tratamiento ortodóncico se lleva a } \\
\text { cabo para corregir las discrepancias dentales } \\
\text { y esqueléticas, algunos autores mostraron } \\
\text { que los resultados del tratamiento de la EMR } \\
\text { también podrían ser eficaces en los problemas } \\
\text { respiratorios naso-respiratorios y trastornos del } \\
\text { sueño de los niños en crecimiento. }\end{array}$ \\
\hline 23 & Conley RS. ${ }^{(70)}$ & $\begin{array}{l}\text { Revisión } \\
\text { narrativa }\end{array}$ & $\begin{array}{l}\text { Presentar las causas comunes } \\
\text { de la apnea obstructiva del } \\
\text { sueño para adultos y población } \\
\text { pediátrica y una revisión de } \\
\text { las formas más exitosas de } \\
\text { tratamiento dental. }\end{array}$ & EMR y DAM & $\begin{array}{l}\text { Resumen de la evidencia actual sobre el } \\
\text { tratamiento de la apnea obstructiva del sueño. }\end{array}$ \\
\hline
\end{tabular}




\section{Bibliografía}

1. Masalán M Patricia, Sequeida Y Julia, Ortiz C Marcela. Sueño en escolares y adolescentes, su importancia y promoción a través de programas educativos. Rev Chil Pediatr. 2013;84(5):554-64.

2. Marcus CL, Brooks LJ, Ward SD, Draper KA, Gozal D, Halbower AC, et al. Diagnosis and management of childhood obstructive sleep apnea syndrome. Pediatrics. 2012; 130(3):e714-e55.

3. Kuhle S, Urschitz MS, Eitner S, Poets CF. Interventions for obstructive sleep apnea in children: A systematic review. Sleep Med Rev. 2009;13(2):123 -31.

4. Davidson Ward SL, Marcus CL. Obstructive sleep apnea in infants and young children. J Clin Neurophysiol. 1996;13(3):198-207.

5. Indriksone I, Jakobsone G. The upper airway dimensions in different sagittal craniofacial patterns: a systematic review. Stomatologija. 2014;16(3):109-17.

6. Katz ES, Mitchell RB, D'Ambrosio CM. Obstructive sleep apnea in infants. Am J Respir Crit Care Med. 2012 Apr 15;185(8):805-16.

7. Pirila-Parkkinen K, Pirttiniemi P, Nieminen P, Tolonen U, Pelttari U, Lopponen H. Dental arch morphology in children with sleep-disordered breathing. Eur $\mathrm{J}$ Orthod. 2009 Apr 1;31(2):160-7.

8. Elso T, Marí José; Brockmann V, Pablo y Zenteno A, Daniel. Consecuencias del síndrome de apnea obstructiva del sueño. Rev Chil Pediatr. 2013,84(2):128-37

9. Iwasaki T, Saitoh I, Takemoto Y, Inada E, Kanomi R, Hayasaki H, et al. Improvement of nasal airway ventilation after rapid maxillary expansion evaluated with computational fluid dynamics. Am J Orthod Dentofacial Orthop. 2012;141(3):269-78. 10. Oliveira De Felippe NL, Da Silveira AC, Viana G, Kusnoto B, Smith B, Evans CA. Relationship between rapid maxillary expansion and nasal cavity size and airway resistance: Short- and long-term effects. Am J Orthod Dentofacial Orthop. 2008;134(3):370-82.

11. Conley RS. Evidence for dental and dental specialty treatment of obstructive sleep apnoea. Part 1: the adult OSA patient and Part 2: the paediatric and adolescent patient: dentistry and OSA. J Oral Rehabil. 2011;38(2):136-56.

12. Machado-Junior A, Zancanella E, Crespo A. Rapid maxillary expansion and obstructive sleep apnea: A review and meta-analysis. Med Oral Patol Oral Cir Bucal. 2016;21(4):e465-9

13. Ngiam J, Cistulli PA. Dental treatment for paediatric obstructive sleep apnea. Paediatr Respir Rev. 2015;16(3):174-81.

14. Camacho M, Chang ET, Song SA, Abdullatif J, Zaghi S, Pirelli $P$, et al. Rapid maxillary expansion for pediatric obstructive sleep apnea: A systematic review and meta-analysis: Rapid Maxillary Expansion for OSA. Laryngoscope. 2017:127(7):1712-9.

15. Huynh NT, Desplats E, Almeida FR. Orthodontics treatments for managing obstructive sleep apnea syndrome in children: A systematic review and metaanalysis. Sleep Med Rev. 2016;25:84-94.

16. Baratieri C, Alves M, de Souza MMG, de Souza Araújo MT, Maia LC. Does rapid maxillary expansion have long-term effects on airway dimensions and breathing? Am J Orthod Dentofacial Orthop. 2011;140(2):146-56.
17. Guilleminault C, Monteyrol P-J, Huynh NT, Pirelli P, Quo S, Li K. Adenotonsillectomy and rapid maxillary distraction in pre-pubertal children, a pilot study. Sleep Breath. 2011:15(2):173 7.

18. Miano S, Rizzoli A, Evangelisti M, Bruni O, Ferri R, Pagani J, et al. NREM sleep instability changes following rapid maxillary expansion in children with obstructive apnea sleep syndrome. Sleep Med. 2009;10(4):471-8.

19. Villa MP, Rizzoli A, Rabasco J, Vitelli O, Pietropaoli N, Cecili M, et al. Rapid maxillary expansion outcomes in treatment of obstructive sleep apnea in children. Sleep Med. 2015;16(6):709-16.

20. Villa MP, Castaldo R, Miano S, Paolino MC, Vitelli O, Tabarrini A, et al. Adenotonsillectomy and orthodontic therapy in pediatric obstructive sleep apnea. Sleep Breath. 2014;18(3):533-9.

21. Villa MP, Miano S, Rizzoli A. Mandibular advancement devices are an alternative and valid treatment for pediatric obstructive sleep apnea syndrome. Sleep Breath. 2012;16(4):971 -6.

22. Katyal V, Pamula Y, Daynes CN, Martin J, Dreyer CW, Kennedy D, et al. Craniofacial and upper airway morphology in pediatric sleep-disordered breathing and changes in quality of life with rapid maxillary expansion. Am J Orthod Dentofacial Orthop. 2013;144(6):860-71.

23. Giannasi LC, Santos IR, Alfaya TA, Bussadori SK, Leitão-Filho FS, de Oliveira LVF. Effect of a rapid maxillary expansion on snoring and sleep in children: a pilot study. Cranio. 2015;33(3):169-73.

24. Pirelli P, Saponara M, Guilleminault C. Rapid maxillary expansion (RME) for pediatric obstructive sleep apnea: a 12-year follow-up. Sleep Med. 2015;16(8):933-5. 25. Kiliç $\mathrm{N}$, Oktay $\mathrm{H}$. Effects of rapid maxillary expansion on nasal breathing and some naso-respiratory and breathing problems in growing children: A literature review. Int J Pediatr Otorhinolaryngol. 2008;72(11):1595-601.

26. Nazarali N, Altalibi M, Nazarali S, Major MP, Flores-Mir C, Major PW. Mandibular advancement appliances for the treatment of paediatric obstructive sleep apnea: a systematic review. Eur J Orthod. 2015;37(6):618-26.

27. Rizk S, Kulbersh VP, Al-Qawasmi R. Changes in the oropharyngeal airway of Class II patients treated with the mandibular anterior repositioning appliance. Angle Orthod. 2016;86(6):955-61.

28. Zhang $\mathrm{C}, \mathrm{He} \mathrm{H}, \mathrm{Ngan} \mathrm{P}$. Effects of twin block appliance on obstructive sleep apnea in children: a preliminary study. Sleep Breath. 2013;17(4):1309-14.

29. Villa MP, Bernkopf E, Pagani J, Broia V, Montesano M, Ronchetti R. Randomized controlled study of an oral jaw-positioning appliance for the treatment of obstructive sleep apnea in children with malocclusion. Am J Respir Crit Care Med. 2002;165(1):123-7.

30. Cozza P, Polimeni A, Ballanti F. A modified monobloc for the treatment of obstructive sleep apnoea in paediatric patients. Eur J Orthod. 2004;26(5):523 -30.

31. Carvalho FR, Lentini-Oliveira DA, Prado LB, Prado GF, Carvalho LB. Oral appliances and functional orthopaedic appliances for obstructive sleep apnoea in children. Cochrane Database Syst Rev. 2016;10:CD005520. 